\title{
Fructosemalabsorption - die andere Seite gesundheitsbewusster Ernährung
}

\section{Fructose Malabsorption - The Other Side of Health-Conscious Nutrition}

Autor

Institut

\section{F.-W. Velten ${ }^{1}$, C. Bayerl ${ }^{2}$}

Klinik für Dermatologie, Venerologie und Allergologie, Medizinische Fakultät Mannheim gGmbH, Ruprecht-Karls-Universität Heidelberg

Klinik für Dermatologie und Allergologie, HSK, Wilhelm Freseniusklinik GmbH, Städtisches Klinikum Wiesbaden

\section{Bibliografie}

Dol $10.1055 / \mathrm{s}-2007-966697$

Online-Publikation: 23. 7. 2007

Akt Dermatol 2007; 33:

373-378 @ Georg Thieme Verlag KG Stuttgart · New York ISSN 0340-2541

Korrespondenzadresse Dr. med. Florian-Walter Velten

Klinik für Dermatologie, Venerologie und Allergologie Medizinische Fakultät Mannheim gGmbH der Ruprecht-Karls Universität Heidelberg

Theodor-Kutzer Ufer 1-3 68167 Mannheim florian.velten@haut.ma. uni-heidelberg.de

\section{Zusammenfassung \\ $\nabla$}

Die Fructosemalabsorption repräsentiert eine weit verbreitete Stoffwechselstörung, welche durch eine Überlastung des enteralen fructosespezifischen GLUT5-Transporters charakterisiert ist. Ins Colon übertretende nicht resorbierte Fructose wird durch Darmbakterien zu kurzkettigen Fettsäuren, Kohlendioxid, Wasserstoff und Methan metabolisiert. Als Folge kommt es zu ei-

\section{Einleitung}

$\nabla$

Gesundheitsbewusste Ernährung ist in den Industrieländern zu einem der wichtigsten gesellschaftlichen Trends geworden. Mit der Intention, der Entwicklung verbreiteter Wohlstandserkrankungen (Adipositas, Typ 2 Diabetes, Herz-Kreislauferkrankungen, Krebs) entgegenzusteuern, haben Biolebensmittel insbesondere nach der BSE-Krise 2000/2001 einen bedeutenden Aufschwung erfahren [1]. Unter der Devise „Man ist was man isst" spielen inzwischen gesundheitliche Werte für 30\% der europäischen Bevölkerung bei der Kaufentscheidung von Lebensmitteln eine bedeutende Rolle [2]. Die Erziehung zur gesunden Lebensführung gewinnt zudem unter den Bedingungen der ansteigenden Kosten im Gesundheitswesen und dem zunehmenden Maß an präventiver Eigenverantwortung bei der Ernährung in Abhängigkeit von sozioökonomischen Faktoren bei einem Teil der Bevölkerung an Bedeutung [3]. Dieser gesundheitsbewusste Bevölkerungsteil verzichtet auf gesättigte, LDL-Cholesterinhaltige tierische Fette, den Einsatz von Kochsalz und glucosehaltige zusätzlich gesüßte Nahrungsmittel. Entsprechend der Empfehlungen von Gesundheitsorganisationen wird ein gesundheitsbewusster Lebensstil mit dem Konsum von „viel“ frischem Obst und Gemüse sowie Vollkornprodukten ohne eine nähere Angabe bezüg- nem Reizdarmsyndrom. Abzugrenzen ist die Fructosemalabsorption von Lebensmittelallergien, der Lactosemaldigestion, der Histaminintoleranz sowie der selten auftretenden hereditären Fructoseintoleranz. Nach der Diagnosestellung mittels des H2-Fructose-Atemtests steht die konsequente diätetische Therapie der Betroffenen durch einen geschulten Ökotrophologen, u.a. mittels einer fructose-reduzierten Kost, im Mittelpunkt der therapeutischen Interventionen.

lich einer Obergrenze gleichgesetzt [4]. Die dabei tolerierten Obergrenzen für Fructose können insbesondere bei Kindern leicht überschritten werden. Diese liegen zwischen dem 1. und dem 10. Lebensjahr bei einer maximal tolerierten täglichen Fructosemenge von 1,0 bis 4,0 g [5]. Schon 1 Apfel mit einem durchschnittlichen Gewicht von $200 \mathrm{~g}$ enthält bereits $11,5 \mathrm{~g}$ reine Fructose. Etwa die Hälfte aller Erwachsenen kann täglich nicht mehr als $25 \mathrm{~g}$ Fructose absorbieren [6]. Etwa 50\% davon zeigen klinische Symptome. So wird nicht absorbierte Fructose in Abhängigkeit von einer nicht-physiologischen Besiedelung des Dünndarms mit Anaerobiern, spätestens jedoch im Dickdarm zu Wasserstoff, $\mathrm{CO}_{2}$ und kurzkettigen Fettsäuren metabolisiert. Als Folge kommt es zu einem Reizdarmsyndrom, das durch Meteorismen, Oberbauchbeschwerden und osmotische Diarrhoen charakterisiert wird. Die diesem Reizdarmsyndrom zugrunde liegende unvollständige Fructoseabsorption wird als Fructosemalabsorption bezeichnet.

\section{Kohlenhydrate in der Ernährung $\checkmark$}

Traditionell konzentriert sich ein Großteil der medizinischen Ernährungsberatung auf Patienten, deren alltägliches Essverhalten mit Erkrankungen aus dem Formenkreis des metabolischen 
Syndroms assoziiert ist. So finden sich auf den Internetseiten der Deutschen Gesellschaft für Ernährung e.V. als Beratungsschwerpunkte der assoziierten Ernährungsberater maßgeblich die Adipositas, Diabetes und Fettstoffwechselstörungen. Eine weitere Fokussierung auf „adipöse Diabetiker“ und zugleich „gesundheitsbewusste Konsumenten“ findet durch industriell hergestellte kohlenhydrathaltige Produkte statt. Die vordergründige Lösung für dieses vermischte Patienten-/Konsumenten-Klientel, nämlich der Ersatz glucosehaltiger Nahrungsmittel durch fructosehaltige Nahrungsmittel, wird unter Beachtung des Auftretens einer möglichen Fructosemalabsorption relativiert. Durch seinen Einsatz in kalorienreduzierten Fertignahrungsmitteln, wie z.B. zur Diabetesprotektion, hat sich der Konsum von Fructose in den letzten Jahrzehnten fast unbemerkt um ein Vielfaches erhöht. Da Fructose im Gegensatz zur Glucose und Saccharose eine stärkere Süßkraft besitzt, hat die Verarbeitung von Fructose in künstlich hergestellten Süßstoffen zu einer enormen Steigerung der täglichen Durchschnittsmenge geführt [7]. Allerdings enthält auch Honig als natürlicher Süßstoff mit durchschnittlich $35 \mathrm{Vol} \%$ besonders hohe Mengen an freier Fructose. Der bedeutsamste Anteil an der alltäglichen Zufuhr von Fructose basiert auf industriell gefertigten Nahrungsmitteln, die nicht ausdrücklich ausgewiesen Fructose-angereicherten Sirup aus Maisstärke (high-fructose corn syrup, HFCS) enthalten. Dabei kommt der Verwendung von HFCS auch eine ökonomische und logistische Bedeutung zu, die durch günstigere Transportmöglichkeiten in Tankwagen zu einer zunehmenden Verdrängung anderer Süßstoffe führt. Zwischen 1970 und 1990 wurde der Konsum an HFCS, der u.a. Limonaden zugesetzt wird, alleine in den USA um $1000 \%$ gesteigert [8]. Die von $50 \%$ aller Erwachsenen täglich maximal tolerierte Fructosemenge wird bereits mit dem Genuss von $30 \mathrm{~g}$ Fructose, enthalten in $500 \mathrm{ml} \mathrm{Li}$ monade, überschritten. Andere Nahrungsmittel, wie z. B. Haselnüsse, Zwiebeln, Möhren, rote Beete, verschiedene Kohlsorten und Sojamehl enthalten mit 2\% bis 5\% ihres Eigengewichtes ebenfalls hohe Fructosemengen.

Ein Blick auf die für die Ernährung natürlicherweise zur Verfügung stehenden Monosaccharide verdeutlicht, dass neben Glucose (Dextrose oder Traubenzucker) und Fructose (Laevulose oder Fruchtzucker) bis auf das Monosaccharid Galactose keine Alternativen existieren. Die vermeintliche Alternative der Galactose wird jedoch dadurch relativiert, dass Galactose im Gegensatz zu Fructose und Glucose in der Natur nicht als freies Monosaccharid, sondern vorwiegend gebunden an Glucose als Disaccharid in Lactose (Milchzucker) vorkommt. Bei Kindern mit ihrer besonders niedrigen Fructosetoleranzschwelle stellt Lactose einen essenziellen Bestandteil der täglichen Zufuhr an Kohlenhydraten dar. Dies wird durch die in jungen Jahren besonders hohe Aktivität der Lactose spaltenden Lactase-PhloritzinHydrolase ( $\beta$-Galactosidase) gewährleistet. Die $\beta$-Galactosidase ist hauptsächlich im Bereich des Jejunums lokalisiert und wird nach der Umstellung der Ernährung von Milch auf andere Nahrungsmittel nach dem Abstillen bei fast allen Tierarten und 70\% der Weltbevölkerung mit starken Schwankungen zwischen einzelnen Kulturkreisen auf 1/10 der ursprünglichen Aktivität herunterreguliert [9]. Häufig kommt es bei den Betroffenen bereits nach dem Konsum von mehr als 1 Glas Milch, entsprechend etwa $200 \mathrm{ml}$, zum Auftreten eines Reizdarmsyndroms. Analog zur Fructosemalabsorption wird Lactose bei einer nicht erfolgten enteralen Absorption durch Anaerobier, entweder im Dickdarm oder bei einer bakteriellen Fehlbesiedelung im Dünndarm, gespalten und führt zu einem Reizdarmsyndrom. Somit sind Er- wachsene zur Deckung ihres täglichen Kalorienbedarfs durch Kohlenhydrate auf andere Disaccharide angewiesen. Dazu zählen die je ein Molekül Glucose und Fructose umfassende Saccharose (Kristallzucker oder Haushaltszucker), die je zwei $\alpha 1,4$-glykosidisch verknüpfte Glucosemoleküle umfassende Maltose (Malzzucker) und die je zwei $\alpha 1,1$-glykosidisch verknüpfte Glucosemoleküle umfassende Trehalose. Saccharose findet sich natürlicherweise in Zuckerrüben, Maltose in verschiedenen Getreidearten und Trehalose hauptsächlich in Pilzen.

Erwachsenen stehen als natürliche Süßstoffe vor allem Monosaccharide zur Verfügung, deren exzessiver Konsum entweder zum metabolischen Syndrom oder zum Reizdarmsyndrom führen kann.

\section{Enterale Absorption von Monosacchariden $\nabla$}

Nach der Ingestion von Kohlenhydraten werden zunächst die komplexeren Oligo- und Polysaccharide durch Amylasen enthalten im Speichel und Pankreassekret gespalten. Im Anschluss werden dann die entstandenen Disaccharide durch Disaccharidasen, die sich luminalwärts gerichtet am apikalen Pol der Enterozyten finden, zu Monosacchariden hydrolysiert ( Tab. 1 und - Abb. 1).

\section{Gastrointestinaler Fructosetransport $\nabla$}

Im Darm wird Fructose langsamer als Glucose resorbiert. Dies liegt am passiven Transport durch spezielle Transportproteine aus der GLUT-Genfamilie (s. Tab.2) [10]. Der im apikalen Anteil der Enterozyten exprimierte GLUT5-Transporter bindet spezifisch Fructose und transportiert diese aus dem Darmlumen in die Enterozyten. Basolateral der Enterozyten wird der GLUT2-Transporter exprimiert, der neben Fructose auch Glucose und Galactose von den Darmzellen in das Blut transportiert ( $\bullet$ Abb. 1). Bei der Fructosemalabsorption kommt es infolge einer erniedrigten GLUT5-Expression zu einer deutlichen Einschränkung des Fructosetransports. Die verminderte GLUT5-Expression kann nicht auf Mutationen innerhalb des GLUT5-Gens zurückgeführt werden [11]. Entsprechend der Ergebnisse von Versuchen an Ratten konnte erst vor Kurzem gezeigt werden, dass die Expression von GLUT5 vom Serumspiegel des Schilddrüsenhormons Trijodthyronin (T3) abhängt [12].

Im Vergleich zu Fructose wird Glucose sekundär-aktiv (SGLT1; sodium-dependent glucose cotransporter 1), also unter Energieverbrauch, in die Zelle gepumpt und unterliegt somit keiner eingeschränkten Transportkapazität. Fructose hingegen fließt ohne Energieaufwand entlang eines Konzentrationsgradienten. Dies führt dazu, dass Fructose niemals vollständig aus der Nahrung aufgenommen wird.

\section{Interaktion von GLUT-Transportern mit Nahrungsmitteln $\nabla$}

Eine Inhibition von GLUT-2, nicht aber von GLUT-5 oder SGTL-1, durch ein Flavonoidgemisch bestehend aus Myricetin, Fisetin, Quercetin und Isoquercetin führt zur kombinierten Inhibition des Glucose- und des Fructosetransports [13,14]. In Lebensmitteln finden sich Flavonoide reichhaltig in Früchten (insbesondere Beeren), Gemüsen (insbesondere Zwiebeln) sowie in Rotwein, 


\begin{tabular}{|c|c|c|c|c|}
\hline Enzym & Alias & Zielmolekül & Primärer Mangel & Sekundärer Mangel \\
\hline Lactase & $\beta$-Galactosidase & Lactose & $\begin{array}{l}\text { physiologisch (selten } \\
\text { kongenitaler Mangel) }\end{array}$ & $\begin{array}{l}\text { durch entz. Darm- } \\
\text { erkrankungen }\end{array}$ \\
\hline Saccharase & Sucrase-Isomaltase & Saccharose & $\begin{array}{l}\text { autosomal rezessiver } \\
\text { Saccharase-Isomaltase- } \\
\text { Mangel }\end{array}$ & $\begin{array}{l}\text { durch entz. Darm- } \\
\text { erkrankungen }\end{array}$ \\
\hline Maltase & $\alpha$-Glucosidase & Maltose & .1 & $\begin{array}{l}\text { Ascarbose, Miglitol } \\
\text { zur Behandlung des } \\
\text { Diabetes mellitus Typ } 2\end{array}$ \\
\hline Trehalase & & Trehalose &.$\%$ & .1 \\
\hline
\end{tabular}

Tab. 1 Disaccharidasen und assoziierte Erkrankungen

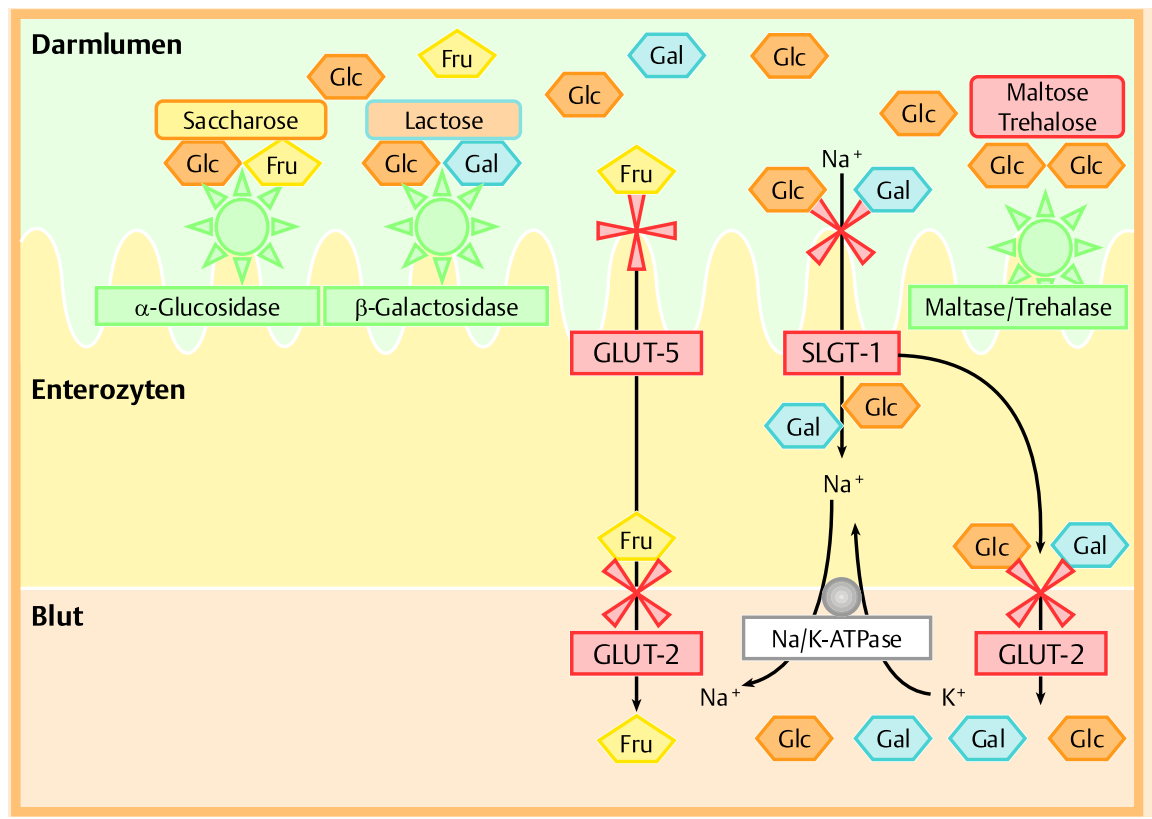

Abb. 1 Enteraler Transport der drei Monosaccharide Fructose (Fru), Glucose (Glc) und Galactose (Gal) durch die Glucosetransporter GLUT-2, GLUT-5 und SLGT-1.

Tee und Arzneipflanzen (Ginkgo-Pflaumen und -Samen, Johanniskraut und Holunder). Ihre gesundheitsfördernden Wirkungen sind zahlreich, angefangen von der antiviralen und antikanzerogenen Wirkung bis hin zur kardioprotektiven Wirkung durch ihre antioxidative Wirkung und eine Hemmung der Thromboxanbildung. Zudem sollen sie mastzellstabilisierende Wirkungen haben und somit die Histaminfreisetzung inhibieren [15]. Darüber hinaus wirkt Quercetin antiinflammatorisch durch seine die Pospholipase A2 als Schrittmacherenzym des Arachidonsäuremetabolismus inhibierende Wirkung.

Quercetin ist als natürlicher Bestandteil von Äpfeln in die Medizin mit der englischen Regel: „an apple a day keeps the doctor away“ eingegangen. Diese Regel macht mit Bezug auf die diätetische Einstellung des Diabetes mellitus und mit Betonung auf den Genuss eines Apfels pro Tag durchaus Sinn. Bei einem Reizdarmsyndrom wird die gesundheitsfördernde Wirkung von Äpfeln und anderen Obst- sowie Gemüsesorten jedoch relativiert. Nicht nur durch die in Äpfeln enthaltenen Flavonoide, sondern auch durch das gerade in unreifen Äpfeln enthaltene Glycosid Phlorizin wird der für den Glucosetransport essenzielle SLGT-1 Transporter inhibiert. Die nach dem Konsum exzessiver Mengen unreifer Äpfel resultierende osmotische Diarrhoe ist auch Personen ohne Reizdarmsyndrom hinlänglich bekannt. Da Phlorizin zugleich zu einer Inhibition der $\beta$-Galactosidase führt, kommt es bei einer Kombination von Äpfeln mit milchhaltigen Produkten zu einer Verstärkung der Symptome. Schließlich wird durch das ebenfalls in Äpfeln enthaltene Flavonoid Quercetin der ohnehin limitierte Fructosetransport über die Inhibition des GLUT-2 Transporters weiter eingeschränkt.

\section{Symptome bei der Fructosemalabsorption $\nabla$}

Fructose erhöht den Blutzuckerspiegel nicht und wird daher besonders in diätetischen Lebensmitteln eingesetzt. Wird die Grenze der durch den GLUT5-Transporter determinierten Grenze der Aufnahmekapazität für Fructose überschritten, kommt es zu gastrointestinalen Beschwerden im Sinne eines Reizdarmsyndroms. Typische Symptome bestehen aus vornehmlich im Bereich des Unterbauches lokalisierten Bauchschmerzen, Meteorismen und zeitweisem Auftreten von Bauchkrämpfen. Daneben klagen die Patienten über weiche Stühle oder rezidivierend auftretende Diarrhoen ohne Blutauflagerungen und ohne Unterbrechung der Nachtruhe. Intermittierend bestehen Perioden mit Obstipationen.

Da eine Fructosemalabsorption mit Resorptionsstörungen der essenziellen Aminosäure Tryptophan einhergeht, kommt es in der Folge zu einer Verminderung der Serotoninsynthese. Neben Depressionen leiden die Patienten unter einem permanenten Süßhunger [16]. Zudem zeigen Patienten mit einer Fructosemalabsorption eine Erniedrigung der Folsäure- und Zinkkonzentration im Serum [17,18]. Schließlich kommt es über Undichtigkeiten der Ileozökalklappe infolge der Druckerhöhung im proximalen Colon zu einer bakteriellen Fehlbesiedelung des Dünndarms, die histologisch als unspezifische Entzündungsreaktion imponiert und zu einer permanenten Immunstimulation führt [19]. 
Tab. 2 Monosaccharidtransporter (MST) der GLUT-Familie (faciliated-diffusion glucose transporter family) und der $\mathrm{Na}^{+}$-abhängigen SGLT-Familie (sodium-dependent glucose cotransporter). Aufgeführt sind ausschließlich humane exprimierte MST, ihre Gewebeverteilung, das transportierte Zielmolekül, die Insulinsensitivität und assoziierte Erkrankungen. HMIT, Myo-Inositol-Transporter

\begin{tabular}{|c|c|c|c|c|c|}
\hline $\begin{array}{l}\text { Transporter } \\
\text { (Protein) }\end{array}$ & Alias & Expressionsort & Zielmolekül & $\begin{array}{l}\text { Insulin- } \\
\text { sensitivität }\end{array}$ & $\begin{array}{l}\text { Assoziierte } \\
\text { Erkrankungen }\end{array}$ \\
\hline GLUT-1 & & $\begin{array}{l}\text { ubiquitäre Verteilung, hpts. ZNS, } \\
\text { Erythrozyten, Tumoren }\end{array}$ & $\begin{array}{l}\text { Glucose (hochaffin), } \\
\text { Galactose }\end{array}$ & ja & $\begin{array}{l}\text { Glucose-Transporter-1- } \\
\text { Defizienz-Syndrom }\end{array}$ \\
\hline GLUT-2 & & $\begin{array}{l}\text { Darmepithel, } \beta \text {-Zellen des } \\
\text { Pankreas, Leber, Niere }\end{array}$ & $\begin{array}{l}\text { Glucose (niedrigaffin), } \\
\text { Galactose, Fructose }\end{array}$ & nein & Fanconi-Bickel-Syndrom \\
\hline GLUT-3 & & $\begin{array}{l}\text { ZNS, Plazenta, Skelettmuskel, } \\
\text { Tumoren }\end{array}$ & $\begin{array}{l}\text { Glucose (hochaffin), } \\
\text { Galactose }\end{array}$ & nein & $\begin{array}{l}\text { Spontanaborte, Störung } \\
\text { des fetalen Wachstums } \\
\text { (experimentell) }\end{array}$ \\
\hline GLUT-4 & & $\begin{array}{l}\text { Fettzellen, Skelettmuskel, Herz } \\
\text { Expression wird mit abnehmen- } \\
\text { dem intrazellulärem Glucose- } \\
\text { gehalt gesteigert } \\
\text { ( } \rightarrow \text { Zuckertransporter) }\end{array}$ & $\begin{array}{l}\text { Glucose (hochaffin), } \\
\text { Galactose }\end{array}$ & ja & $\begin{array}{l}\text { nicht-insulinpflichtiger } \\
\text { Diabetes mellitus } \\
\text { (Insulinresistenz) }\end{array}$ \\
\hline GLUT-5 & & $\begin{array}{l}\text { Dünndarm, Spermatozoen, } \\
\text { Niere, Skelettmuskel, } \\
\text { Erythrozyten }\end{array}$ & Fructose & nein & Fructosemalabsorption \\
\hline GLUT-6 & GLUT-9 & Milz, Leukozyten, ZNS & Glucose & nein & unbekannt \\
\hline GLUT-7 & & $\begin{array}{l}\text { Dünndarm, Leber } \\
\text { Transport von aus der } \\
\text { Gluconeogenese entstandenen } \\
\text { Glucose ins Blut }\end{array}$ & Glucose, Fructose & unbekannt & unbekannt \\
\hline GLUT-8 & GLUT-X1 & Hoden, Skelettmuskel, ZNS & Glucose & nein & unbekannt \\
\hline GLUT-9 & GLUT-X & Niere, Leber & $?$ & unbekannt & unbekannt \\
\hline GLUT-10 & & Leber, Pankreas & Glucose & nein & unbekannt \\
\hline GLUT-11 & GLUT-10 & Herz, Muskel & $\begin{array}{l}\text { Glucose, Fructose } \\
\text { (niedrigaffin) }\end{array}$ & nein & unbekannt \\
\hline GLUT-12 & GLUT-8 & $\begin{array}{l}\text { Skelettmuskel, Herz, } \\
\text { Prostata }\end{array}$ & $?$ & ja & unbekannt \\
\hline HMIT & & ZNS & Myo-Inositol & unbekannt & unbekannt \\
\hline SGLT-1 & & $\begin{array}{l}\text { hpts. Dünndarm, gering in Niere, } \\
\text { Lunge und Leber }\end{array}$ & $\begin{array}{l}\text { Glucose (hochaffin), } \\
\text { Galactose }\end{array}$ & nein & $\begin{array}{l}\text { Glucose-Galactose- } \\
\text { Malabsorptionssyndrom }\end{array}$ \\
\hline SGLT-2 & & Niere & Glucose (niedrigaffin) & nein & renale Glucosurie \\
\hline SGLT-3 & & Niere, Leber, Milz, Muskel & Glucose (niedrigaffin) & nein & unbekannt \\
\hline SGLT-4 & & Dünndarm, Niere & Glucose & nein & unbekannt \\
\hline
\end{tabular}

\section{Differenzialdiagnosen der Fructosemalabsorption $\boldsymbol{\nabla}$}

\section{Nahrungsmittelallergien}

In Europa und den USA leiden Schätzungen zufolge etwa 6-8\% der Kinder und 3-4\% der Erwachsenen an einer IgE-vermittelten Nahrungsmittelallergie [20-22]. In Abgrenzung zur Fructosemalabsorption können die Betroffenen über die gastrointestinalen Symptome eines Reizdarmsyndroms hinaus systemische, IgE-vermittelte Reaktionen, von der Urtikaria über das Angioödem bis hin zum anaphylaktischen Schock aufweisen. Nahrungsmittelallergien lassen sich in nicht-pollenassoziierte und pollenassoziierte Allergien unterteilen. Erstere treten vornehmlich vor dem 5. Lebensjahr auf und betreffen im Wesentlichen die nicht fructosehaltigen Nahrungsmittel Milch-, Ei-, Fischund Erdnussproteine. Eine Abgrenzung von pollenassoziierten Nahrungsmittelallergien gegenüber der Fructosemalabsorption ist schwieriger, da beide Gruppen fructosehaltige Obst- und Gemüsesorten sowie Nüsse betreffen. Pollenassoziierte Nahrungsmittelallergien treten meist nach dem 7. Lebensjahr auf, stehen in enger Beziehung zu Sensibilisierungen des Respirationstraktes und führen fast immer zum Auftreten eines oralen Allergiesyndroms.

Unter physiologischen Bedingungen kommt es infolge der Präsenz verschiedener Populationen regulatorischer T-Zellen bei einer intakten intestinalen Mucosabarriere sowie dem Vorhandensein der eine Th1-Immunantwort forcierenden kommensalen Darmflora zur Ausbildung einer oralen Toleranz gegenüber Nahrungsmittelproteinen. Eine Zerstörung der intestinalen Mucosabarriere durch chronische Entzündungen sowie eine bakterielle Fehlbesiedelung führen zu einer Durchbrechung der oralen Toleranz und somit zur Ausbildung von Nahrungsmittelallergien [23]. Eine Assoziation einer lange bestehenden Fructosemalabsorption zum Auftreten von Nahrungsmittelallergien ist somit denkbar. Die genauen pathophysiologischen Mechanismen, welche zumindest zu einer umschriebenen Dominanz einer die Produktion spezifischer gegen Nahrungsmittelallergene gerichteter IgE-Antikörper führenden Th2-Immunantwort bedingen wird jedoch weder bei der Fructosemalabsorption noch bei anderen chronischen Darmentzündungen, wie z. B. der Zöliakie, verstanden.

\section{Lactosemaldigestion}

Die Lactosemaldigestion kann auf einen Mangel des Verdauungsenzyms Lactase zurückgeführt werden. Nach dem Genuss einer die Restaktivität der Lactase überschreitenden Menge an Lactose kommt es infolge der Vergärung überschüssiger Lactose durch Darmbakterien zum Auftreten eines Reizdarmsyndroms. Die klinischen Symptome bei der Lactosemaldigestion unter- 


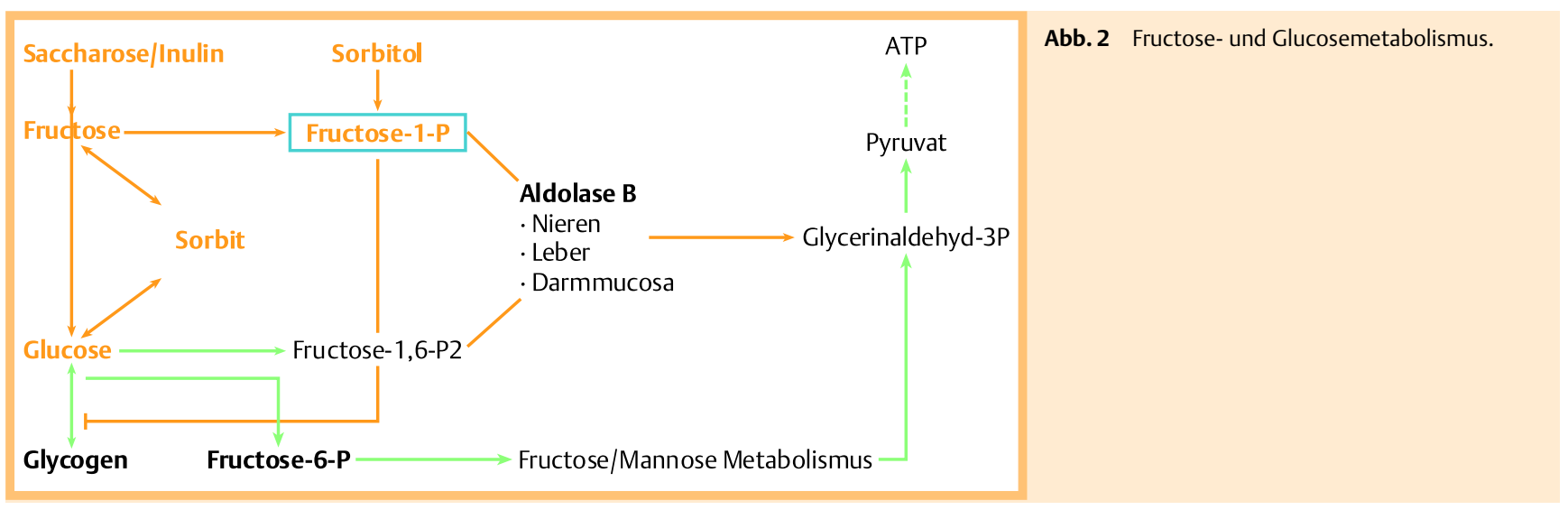

scheiden sich somit nicht von denjenigen einer Fructosemalabsorption. Eine Differenzierung beider Unverträglichkeitsreaktionen wird durch eine genaue Erhebung einer Nahrungsmittelanamnese sowie mittels Durchführung eines Lactose-H2-Atemtests ermöglicht.

\section{Histaminintoleranz}

Die Histaminintoleranz resultiert aus einem Ungleichgewicht aus sich akkumulierendem endogenen und exogenen über die Nahrung aufgenommenen Histamins sowie einer unzureichenden Kapazität Histamin-abbauender Enzyme, insbesondere der Diaminooxidase (DAO). Neben gastrointestinalen Symptomen zeigen die Betroffenen nach dem Genuss von histaminreichen Speisen (insbesondere mikrobiell produzierte oder kontaminierte Nahrungsmittel wie z. B. lang gereifter Käse, Wein, Sauerkraut, Fisch und Salami) und histaminliberierender oder DAO-blockierender Speisen bzw. Medikamente (u.a. Tomaten, Erdbeeren, Röntgen-Kontrastmittel, Lokalanästhetika, Analgetika) Kopfschmerzen, rhinitische Beschwerden, einen Flush und kardiale Symptome im Sinne von Tachyarrhythmien. In Abgrenzung zur Fructosemalabsorption können Antihistaminika oder die Substitution der Diaminooxidase zu einer Besserung der Beschwerdesymptomatik führen.

\section{Hereditäre Fructoseintoleranz}

Nach seiner Absorption wird Fructose durch das Enzym Fructokinase in Fructose-1-phosphat und anschließend durch Fructose-1-phosphat-Aldolase (Aldolase B) zu Glycerinaldehyd und Dihydroxyacetonphosphat umgewandelt. Neben der Darmmucosa ist die Aldolase B auch in den Nieren und in der Leber lokalisiert. Bei der seltenen hereditären Fructoseintoleranz (Inzidenz: $1: 20000)$ kann Fructose-1-Phosphat infolge eines autosomal rezessiven Defektes des Aldolase-B-Gens nicht weiter metabolisiert werden ( $\bullet$ Abb. 2). Neben einer ausbleibenden Energiegewinnung aus Fructose selbst inhibiert Fructose-1-Phosphat auch die Glycogenolyse und führt somit zu nicht durch Glucagon zu beeinflussenden Hypoglycämien. Es kommt somit zur absurden Situation eines Blutzuckerabfalls nach dem Genuss fructosereicher, äußerst süßer Nahrungsmittel. Bei lang andauernder Fructosezufuhr führt die hereditäre Fructoseintoleranz über eine renal-tubuläre Azidose zu einem renalen Fanconi-Syndrom, dass durch einen Phosphat-, Glucose und einen Aminosäurenverlust sowie einer verminderten Hydroxilierung von 15-Hydroxy-Vitamin D3 charakterisiert ist. Neben der nephrotoxischen Wirkung führt Fructose-1-Phosphat über eine hepatotoxische Wirkung zur Leberzirrhose.

\section{Diagnose der Fructosemalabsorption $\nabla$}

Beim Vorliegen eines Reizdarmsyndroms mit rezidivierenden nicht-nächtlichen Diarrhoen, Meteorismen und abdominalen Krämpfen sollte erfragt werden, ob es zu einer Verstärkung dieser Symptome durch besonders fructosereiche Nahrungsmittel (Honig, Nüsse, Ketchup, Frucht- und Gemüsesäfte) kommt. Im Anschluss sollte zur Objektivierung einer Fructosemalabsorption eine Bestimmung der H2-Exhalationswerte beim nüchternen Patienten nach einer oralen Belastung mit $25 \mathrm{~g}$ Fructose erfolgen. Die Messung erfolgt in halbstündigen Abständen über mindestens 2 Stunden. Ein Anstieg der H2-Exhalationswerte um 20 ppm über den Ausgangswert kann als Fructosemalabsorption gewertet werden. Einzig eine bakterielle Fehlbesiedelung des oberen Gastrointestinaltraktes, die eine vorzeitige Metabolisierung intraluminaler Fructose verursacht, muss bei pathologisch erhöhten H2-Exhalationswerten differenzialdiagnostisch beachtet werden. Daher sollte sich in diesen Fällen an den Fructose-Atemtest ein Glucose-Atemtest anschließen.

Zudem sollte bei einer im Atemtest nachgewiesenen Fructosemalabsorption eine Bestimmung der Folsäure, des Zinks und von Vitamin C erfolgen. Bei einer positiven Anamnese sollte zudem mittels der Prick- und Scratchtestung sowie ggf. durch die Bestimmung spezifischer IgE-Antikörper ein Ausschluss von Nahrungsmittel(additiva)-Intoleranzen erfolgen.

\section{Therapie der Fructosemalabsorption $\nabla$}

Eine Therapie der Fructosemalabsorption sollte in Zusammenarbeit mit einem für dieses Krankheitsbild geschulten Ökotrophologen erfolgen. Zur Orientierung dienen die in $\bullet$ Tab. 3 aufgeführten Nahrungsmittel. Das Ziel der diätetischen Interventionen liegt in der Reduktion der mit der Nahrung aufgenommenen Fructose und von Sorbit auf ein individuell verträgliches Maß. Sorbit findet sich in vielen Obstsorten, Bier und als Zuckeraustauschstoff in Diabetikerprodukten sowie „zuckerfreien“ Kaugummies. Durch eine Zufuhr von Sorbit wird die gastrointestinale Fructoseresorption durch eine Hemmung des GLUT-5 Transporters weiter vermindert. Demgegenüber wirkt sich die gleichzeitige Zufuhr von Glucose, entweder als Monosaccharid oder in Kombination mit Fructose als Disaccharid (Saccharose) fördernd auf die Fructosetransportkapazität aus. Durch den gleichzeitigen Verzehr von fructosereichem Obst und Traubenzucker kann insbesondere Vitamin-C-Mangelzuständen vorgebeugt werden. Die bei der Fructosemalabsorption erniedrigten Serumspiegel für 
Tab. 3 Fructose-, Saccharose-, Sorbit-, Sorbitol-, Inulin- und Xylit-freie Ernährung

\begin{tabular}{|c|c|c|}
\hline & Verbotene Lebensmittel & Erlaubte Lebensmittel \\
\hline Süßigkeiten & $\begin{array}{l}\text { Honig, Marmeladen, Nutella, Süßigkeiten aller Art, } \\
\text { Speiseeis, „zuckerfreie“ Kaugummis }\end{array}$ & Traubenzucker, Fa. Sarotti-Kakao \\
\hline Nährmittel & Backwaren mit Zucker, Brot, Semmeln, Vollkornprodukte & $\begin{array}{l}\text { Weizenmehl Typ } 480 \text { mit wenig Roggenanteil, Mais, Grieß, } \\
\text { Reis, Hirse }\end{array}$ \\
\hline Milchprodukte & Milchprodukte gesüßt/mit Früchten, Käseaufstriche & $\begin{array}{l}\text { Butter, Buttermilch, Eier, Schafsmilchprodukte, Käse ohne } \\
\text { Zusatz }\end{array}$ \\
\hline Fleisch/Fisch & Wurst/Fleisch mit Zusatzstoffen (als Salate), Fischkonserven & Fleisch/Fisch frisch \\
\hline Knabbereien & Zwieback, alle Nüsse, Mandeln, Kastanien & Dinkel-Soletti, Reiswaffeln \\
\hline Gewürze/Essig/Öl & Ketchup, Gewürzmischungen, Essig, Knoblauch & $\begin{array}{l}\text { Dill, Basilikum, Petersilie, Safran, Thymian, Salz, Pfeffer, } \\
\text { Olivenöl }\end{array}$ \\
\hline Getränke & Frucht-/Gemüsesäfte (Tee), Weisswein, Sekt, Bier (Sorbit) & $\begin{array}{l}\text { Leitungswasser, Kaffee, Schwarztee, Kräutertee, } \\
\text { max. } 1 / 8 \text { I herben Rotwein }\end{array}$ \\
\hline Obst/Gemüse & $\begin{array}{l}\text { Früchte, Soja, Zwiebel, viele Gemüsesorten, Kohl und } \\
\text { grüne Bohnen zu Beginn der Diät }\end{array}$ & $\begin{array}{l}\text { Zitronensaft, evtl. Bananen, weiße Kartoffeln, Kopfsalat, } \\
\text { Spinat, Spargel }\end{array}$ \\
\hline
\end{tabular}

Zink und Folsäure sollten substituiert werden. Entsprechend der Richtlinien des gemeinsamen Bundesausschusses über die Verordnung von Arzneimitteln in der vertragsärztlichen Versorgung (26.09.2004; Pkt. 16.4.39) ist die Substitution bei nachgewiesenem Mangel erstattungsfähig.

\section{Abstract}

\section{Fructose Malabsorption - The Other Side of Health-Conscious Nutrition}

$\nabla$

Fructose malabsorption or dietary fructose intolerance represent a widespread metabolic disorder caused by an overload of the enteral fructose-specific GLUT5 transporter. Fructose that reaches the colon is metabolized by colonic bacteria to short chain fatty acids and the gases carbon dioxide, hydrogen and methane which lead to irritable bowel syndrome. Fructose malabsorption is not to be confused with food allergies, lactose maldigestion, histamine intolerance and hereditary fructose intolerance. Medical tests require a fructose-based hydrogen breath test for a clinical diagnosis. Depending on the individual threshold for fructose, an appropriate fructose-reduced diet should be initiated by a trained ecotrophologist.

\section{Literatur}

1 Lorenz S. Natur und Politik der Biolebensmittelwahl. Kulturelle Orientierung im Konsumalltag. Berlin: Wissenschaftlicher Verlag Berlin, 2005

2 Lennernas $M$, Fjellstrom C, Becker $W$ et al. Influences on food choice perceived to be important by nationally-representative samples of adults in the European Union. Eur J Clin Nutr. 1997; 51: S8 - S15

3 Deshmukh-Taskar P, Nicklas TA, Yang SJ et al. Does food group consumption vary by differences in socioeconomic, demographic, and lifestyle factors in young adults? The bogalusa heart study. J Am Diet Assoc 2007; 107: 223 - 234

4 Lichtenstein AH, Appel LJ, Brands $M$ et al. Diet and lifestyle recommendations revision 2006: a scientific statement from the American Heart Association Nutrition Committee. Circulation 2006; 114: 82 - 96

5 Wachtel $U$, Hilgart $R$. Ernährung und Diätetik in Pädiatrie und Jugendmedizin. Stuttgart: ed. G. T. V, 1995

6 Gibson PR, Newnham E, Barrett JS et al. Review article: fructose malabsorption and the bigger picture. Aliment Pharmacol Ther 2007; 25: $349-363$
7 Beyer PL, Caviar EM, McCallum RW. Fructose intake at current levels in the United States may cause gastrointestinal distress in normal adults. J Am Diet Assoc 2005; 105: 1559- 1566

8 Bray GA, Nielsen SJ, Popkin BM. Consumption of high-fructose corn syrup in beverages may play a role in the epidemic of obesity. Am J Clin Nutr 2004; 79: 537-543

9 Vesa TH, Marteau P, Korpela R. Lactose intolerance. J Am Coll Nutr 2000; 19: 165S-175S

10 Mueckler M. Facilitative glucose transporters. Eur J Biochem 1994; 219: $713-725$

11 Wasserman D, Hoekstra JH, Tolia $V$ et al. Molecular analysis of the fructose transporter gene (GLUT5) in isolated fructose malabsorption. J Clin Invest 1996; 98: 2398-2402

12 Mochizuki K, Sakaguchi N, Goda T. Triiodothyronine (T(3)) and Fructose Coordinately Enhance Expression of the GLUT5 Gene in the Small Intestine of Rats during Weaning Period. Biosci Biotechnol Biochem 2007; 71: $1345-1347$

13 Song J, Kwon O, Chen S et al. Flavonoid inhibition of sodium-dependent vitamin C transporter 1 (SVCT1) and glucose transporter isoform 2 (GLUT2), intestinal transporters for vitamin C and Glucose. J Biol Chem 2002; 277: 15252 - 15260

14 Kwon 0, Eck P, Chen $S$ et al. Inhibition of the intestinal glucose transporter GLUT2 by flavonoids. Faseb J 2007; 21: 366 - 377

15 Yao LH, Jiang YM, Shi J et al. Flavonoids in food and their health benefits. Plant Foods Hum Nutr 2004; 59: $113-122$

16 Ledochowski M, Widner B, Propst-Braunsteiner T et al. Fructose malabsorption is associated with decreased plasma tryptophan. Adv Exp Med Biol 1999; 467: 73-78

17 Ledochowski $M$, Uberall $F$, Propst $T$ et al. Fructose malabsorption is associated with lower plasma folic acid concentrations in middleaged subjects. Clin Chem 1999; 45: 2013 - 2014

18 Ledochowski M, Widner B, Murr C et al. Decreased serum zinc in fructose malabsorbers. Clin Chem 2001; 47: 745 - 747

19 Nucera G, Gabrielli M, Lupascu A et al. Abnormal breath tests to lactose, fructose and sorbitol in irritable bowel syndrome may be explained by small intestinal bacterial overgrowth. Aliment Pharmacol Ther 2005; 21: $1391-1395$

20 Moneret-Vautrin AD. Gastrointestinal allergy in adults. Eur J Gastroenterol Hepatol 2005; 17: $1293-1297$

21 Rance F, Grandmottet X, Grandjean $H$. Prevalence and main characteristics of schoolchildren diagnosed with food allergies in France. Clin Exp Allergy 2005; 35: 167 - 172

22 Sicherer SH, Sampson HA. 9. Food allergy. J Allergy Clin Immunol 2006; 117: S470-S475

23 Mansueto P, Montalto G, Pacor ML et al. Food allergy in gastroenterologic diseases: Review of literature. World J Gastroenterol 2006; 12: $7744-7752$ 